\title{
A Software Toolkit to Study Systematic Uncertainties of the Physics Models of the Geant4 Simulation Package
}

\author{
Krzysztof Genser, Robert Hatcher, Gabriel Perdue, Hans Wenzel, Julia Yarba* \\ Fermi National Accelerator Laboratory, Batavia, IL (USA) \\ E-mail: varba jefnal.gov
}

\section{Michael Kelsey, Dennis H. Wright}

SLAC National Accelerator Laboratory, Stanford, CA (USA)

\begin{abstract}
The Geant 4 toolkit is used to model interactions between particles and matter. Geant 4 employs a set of validated physics models that span a wide range of interaction energies. These models are tuned to cover a large variety of possible applications. This raises the critical question of what uncertainties are associated with the Geant 4 physics model, or group of models, involved in a simulation project. To address the challenge, we have designed and implemented a comprehensive, modular, user-friendly software toolkit that allows the variation of one or more parameters of one or more Geant 4 physics models involved in simulation studies. It also enables analysis of multiple variants of the resulting physics observables of interest in order to estimate the uncertainties associated with the simulation model choices. Key functionalities of the toolkit are presented in this paper and are illustrated with selected results.
\end{abstract}

38th International Conference on High Energy Physics

3-10 August 2016

Chicago, USA

* Speaker. 


\section{Introduction}

Geant4 [四] is the leading detector simulation toolkit used in high energy physics to design detectors and to optimization calibration and reconstruction software. It offers a set of carefully validated physics models to simulate interactions of particles with matter accross a wide range of interaction energies. These models, especially the hadronic ones, rely largely on directly measured cross-sections and phenomenological predictions with physically motivated parameters estimated by theoretical calculation or measurement. Because these models are tuned to cover a very wide range of possible simulation tasks, they may not always be optimized for a given process or a given material. This raises a question of how sensitive Geant 4 predictions are to varying one or more parameters of a model or a group of models involved in a simulation project. To address this, the Geant 4 collaboration started a dedicated effort to develop a software toolkit to study how variations of model parameters affect simulated observables and to explore the associated errors in a convenient, comprehensive, run-time configurable, and documented way.

Note that there are two tasks here - changing the central value of a given parameter and estimating uncertainties for a given value of the parameters; it is important to be able to do these tasks independently. The primary goal is to estimate the physically meaningfull range of validity of the different model parameters and to determine their optimal values and uncertainties from a global fit to relevant data. Model parameters exposed to the users will be thoroughly documented.

\section{Toolkit Features}

We have designed and implemented a comprehensive, modular, user-friendly software package that allows users to vary parameters of the Geant 4 physics models involved in the simulation studies, and to analyze multiple variants of the resulting physics observables simultaneously. Geant4.10.1.patch02 or later is required to operate this software. The toolkit is based on a modern event-processing framework [ [ $]$ ] and offers a variety of features, such as a user-friendly model configuration API, flexible run-time configurable workflow, comprehensive bookkeeping, analysis of multiple variants of the resulting physics observables of interest, and an extensible design. The toolkit components and the workflow are schematically presented in Figure 1. The key features are listed here.

The Geant 4 model parameters API enables users to change parameters of one or several models. It also allows users to restore default settings and/or to print current settings. Among other features, this component ensures that the application is in a proper Geant 4 state for the parameters to propagate to the model.

The Geant 4 application module can be configured at run time with a flexible geometry and sensitive detector setup in GDML [3] format and a of physics list [四] of user's choice. Events simulated by different Geant 4 variants can be easily added to the same output file for further analysis.

The single interaction simulation module allows the modeling of a single particle-nucleus interaction. The incident particle and target nucleus are chosen at run time. Multiple instances of this module can run in the same job. 
A group of example analysis modules provides an extensible collection of run-time configurable components. Multiple analysis modules can be included in the same job. Analysis can be done in the same job as simulation or in a separate step, if desired.

Example end-of-the-job summary analysis module shows how to employ the RooMUHistos package [1] to perform end-of-job combinations of compatible variants for further analysis. Multiple modules of this type can run in the same job, if desired.

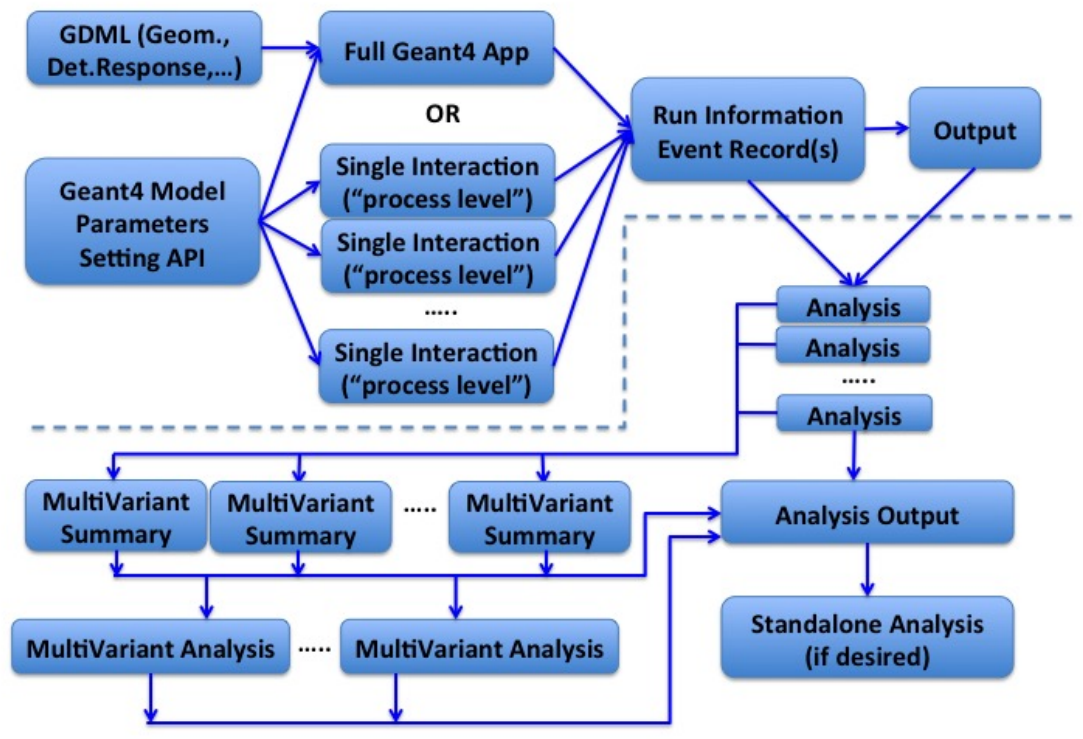

Figure 1: Software components and workflow.

\section{Preliminary Results}

For the first use-case study, we explored the sensitivity of the Bertini Cascade model [目] to variations of its parameters. The toolkit has been used to study effects of varying selected parameters of the Bertini cascade on such simulated observables as energy deposit in the LArIAT [ $\square$ ] Liquid Argon detector and hadron production in hadron-nucleus interactions. The simulation of the LArIAT Liquid Argon Detector uses the QGSP_FTFP_BERT physics list [U, 团] that includes the Bertini Cascade model covering the $0-9.9 \mathrm{GeV}$ energy range. Predictions using the default values of Geant4 Bertini parameters are compared with results obtained with several alternative settings of these parameters within a physically meaningful range. In this study the varied parameters were the nuclear radius and the "internal" hadron-nucleon cross-section that an incident particle sees as it travels through the target nucleus ("Radius Scale" and "XSec Scale" in Figures 2 and 3). It should be noted that some of the parameter shifts have been made especially large in this study to make the effects easier to see. Results of modeling hadron-nucleus interactions with different settings of the Bertini Cascade model parameters are compared with experimental data from the HARP [ [ $\mathbb{Q}, \mathbf{Q}]$ experiment. Preliminary results are shown in Figure 2 and Figure 3. 


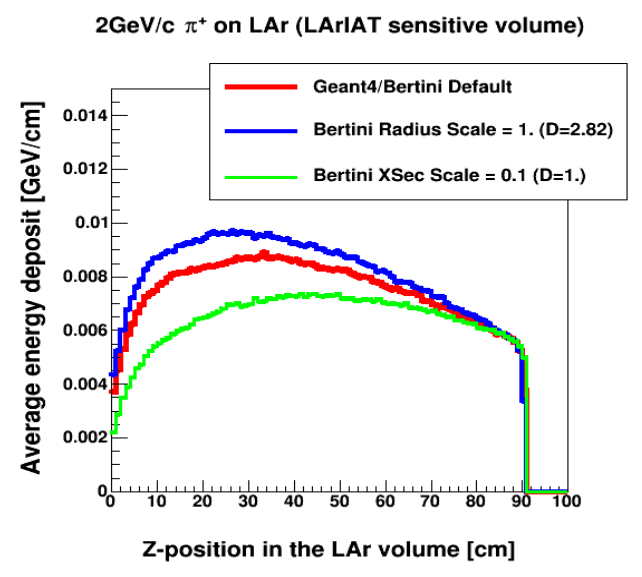

Figure 2: Longitudinal profile of a simulated hadronic shower induced by $2 \mathrm{GeV} / \mathrm{c} \pi^{+}$incident on Liquid Argon detector volume of the LArIAT setup [四].

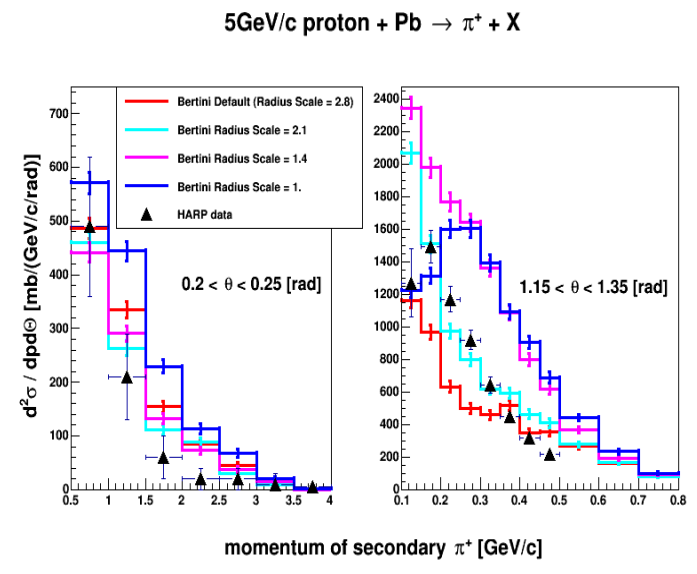

Figure 3: Production cross section of $\pi^{+}$in protonLead interations at $5 \mathrm{GeV} / \mathrm{c}$ shown as a function of $\pi^{+}$momentum in different intervals of the polar angle, as simulated by Bertini Cascade model.

\section{Summary}

In response to requests from the user community, we are developing a software toolkit to explore the impact of varying Geant 4 model parameters on the simulated physics results. The toolkit was used to study the effects of varying Geant4 hadronic model parameters on simulated observables, with the Bertini cascade model as the first use-case. Selected results are included in this presentation for illustration. Further development of the toolkit and inclusion of other key Geant 4 models in the study are planned for the near future.

\section{References}

[1] J. Allison et al., Recent Development in Geant4, Nucl. Instrum. Meth. A835 (2016) 186.

[2] http://art.fnal.gov/.

[3] R. Chytracek et al., Geometry Description Markup Language for Physics Simulation and Analysis Applications, IEEETrans. Nucl. Sci. 53 (2006) 2892.

[4] http://geant4.web.cern.ch/geant4/UserDocumentation/UsersGuides/ForApplicationDeveloper/html

[5] https://github.com/ManyUniverseAna/RooMUHistos/.

[6] D.H. Wright, M.H. Kelsey, The Geant4 Bertini Cascade, Nucl.Instrum.Meth. A804 (2015) 175.

[7] http://lariat.fnal.gov/.

[8] M.G. Catanesi et al., Large-angle production of charged pions with 3-12.9 GeV/c incident protons on nuclear targets, Phys.Rev. C77 (2008) 05520.

[9] M. Apollonio et al., Forward production of charged pions with incident protons on nuclear targets at the CERN PS, Phys.Rev. C80 (2009) 035208. 